\title{
Stability of Hyaluronan-Pectic Gel Particles in the Conditions of the Artificial Gastrointestinal Environment
}

\author{
Elena A. Mikhailova ${ }^{1 *}$; Anatoly K. Melekhin ${ }^{1}$; \\ Vladimir A. Belyy, $\mathrm{PhD}^{2}$; Anatoly A. Shubakov, $\mathrm{PhD}^{1}$ \\ IInstitute of Physiology, Komi Science Centre, Ural Branch of the RAS \\ ${ }^{2}$ Institute of Chemistry, Komi Science Centre, Ural Branch of the RAS \\ Syktyvkar, Komi Republic, the Russian Federation
}

\begin{abstract}
Spherical hyaluronan-pectic gel particles (HPGPs) from hyaluronic acid (HA) and low-methyl esterified pectins of callus cultures of tansy, duckweed, campion and commercial apple pectin were obtained by the method of ionotropic gelation in the presence of calcium ions. We investigated the morphology, swelling and degradation of the obtained HPGPs in the conditions of a simulated gastrointestinal environment and established that the greatest stability in the artificial environment of the digestive tract is achieved with HPGPs obtained from the pectin of tansy callus cultures. HPGPs can be used as potential carriers for drug delivery systems in parts of the small and large intestine.(International Journal of Biomedicine. 2017;7(4):310-314.)
\end{abstract}

Key Words: hyaluronic acid $\bullet$ pectin $\bullet$ callus culture $\bullet$ gel particles $\bullet$ gastrointestinal environment

\section{Abbreviations}

DDS, drug delivery systems; GIT, gastrointestinal tract; GLC, gas-liquid chromatography; HA, hyaluronic acid; HPGPs, hyaluronan-pectic gel particles.

\section{Introduction}

Pectins are natural water-soluble, non-toxic, biodegradable and biocompatible polysaccharides of plant origin exhibiting high physiological activity. Pectic polysaccharides, the main carbohydrate chain of which is represented by 1,4 -linked residues of $\alpha$-Dgalactopyranosyluronic acid, are capable of gelling. In recent years, the pectins in the form of spherical particles have been studied for use in drug delivery systems in the body (DDS - drug delivery systems). ${ }^{(1-3)}$ Calcium-pectic gel particles delay drug release in the upper gastrointestinal tract and release it as a result of the degradation of particles by pectic enzymes of the colon. ${ }^{(4)} \mathrm{HA}$ is a biocompatible immunoneutral

*Corresponding author: Elena A. Mikhailova. Department of Molecular Immunology and Biotechnology, Institute of Physiology, Komi Science Centre, Ural Branch of the RAS. Syktyvkar, the Russian Federation. E-mail: elena_elkina@mail.ru mucopolysaccharide whose macromolecules consist of disaccharide units, the components of which are N-acetyl-Dglucosamine and D-glucuronic acid, interconnected by $\beta-1 \rightarrow 4$ and $\beta-1 \rightarrow 3$ bonds. HA in the form of salts, polyelectrolyte complexes or mixtures with other substances of polymeric or other natures has been used more widely in recent years in aesthetic medicine, the treatment of joint diseases, and tissue engineering, as an adhesion barrier to prevent the formation of adhesions in surgery. ${ }^{(5,6)}$ However, the biomedical use of HA is hampered by its short life span and insufficient mechanical strength in the aquatic environment.

To increase the strength and efficiency of natural polymers as carriers of drugs, they can be conjugated. ${ }^{(7)}$ Thus, some reports have demonstrated the preparation of gel particles from HA and chitosan. ${ }^{(8)}$ We have obtained the complex hydrogel particles from $\mathrm{HA}$ and pectins of callus cultures for the first time.

The purpose of this work was to obtain HPGPs and to study their morphology and the process of their degradation 
under conditions of an artificial gastroenteric environment as potential systems for the targeted delivery of drugs to the small and large intestines.

\section{Materials and Methods}

\section{Objects of the study and conditions of cultivation of callus} cultures

In this work, we used HA from cock-crests (SigmaAldrich, United Kingdom) with the molecular mass of $>300 \mathrm{kDa}$, commercial apple pectin AU-701 with a degree of methoxylation of 36\%-44\% and with the molecular weight of $406 \mathrm{kDa}$ (AP, Herbstreith \& Fox KG, Germany, low-methyl esterified pectins (6\%-22\%) from callus cultures of tansy Tanacetum vulgare $\mathrm{L}$. (TVC), duckweed Lemna minor L. (LMC), campion Silene vulgaris (M.) G. (SVC) with a molecular mass of $>300 \mathrm{kDa}$, and calcium chloride $\left(\mathrm{CaCl}_{2}\right.$, Sigma, USA).

Callus cultures of the bladder campion, tansy and common duckweed were grown on modified Murashige and Skoog agar medium. ${ }^{(9)}$ The bladder campion and common duckweed CC were cultivated with $1.0 \mathrm{mg} / 1$ of 2,4-dichlorophenoxyacetic acid (2,4-D) and $0.5 \mathrm{mg} / \mathrm{l}$ of 6-benzylaminopurine (BAP) added to the medium. The tansy callus was cultivated with the addition of 2,4-D $(1.5 \mathrm{mg} / \mathrm{l})+\mathrm{BAP}(0.5 \mathrm{mg} / \mathrm{l})$. The calluses were subcultured with an interval of 21 days (campion) and 28 days (tansy and duckweed) at the temperature of $26 \pm 1^{\circ} \mathrm{C}$ in the dark. The callus tissue was frozen at the end of the cultivation. ${ }^{(10,11)}$

\section{Isolation of polysaccharides}

Before the isolation of polysaccharides, the biomass was degraded by a single freeze-thaw cycle. The extraction of biomass was carried out with distilled water at $50^{\circ} \mathrm{C}$ for 6 hours, the raw material:solution ratio was 1:10. The biomass was then separated from the extract and treated with HA solution ( $\mathrm{pH} 4.0)$ with a 1:10 ratio of raw material:solution at $50^{\circ} \mathrm{C}$ for 3 hours to make the pectin substances water soluble. Then, the pectins were extracted by an $0.7 \%$ aqueous solution of ammonium oxalate with a 1:10 ratio of raw material:solution at $68-70^{\circ} \mathrm{C}$ for 6 hours. The extraction was carried out in the digester VK-V-100 (Russia).

The resultant extract was separated from the plant mass by centrifugation $\left(5^{\circ} \mathrm{C}, 9500 \mathrm{rpm}, 2 \mathrm{~h}\right)$ with a continuous-flow centrifuge (Beckman Coulter Avanti J-25I with cooling and a flow-through rotor JCF-Z) and sequentially concentrated with simultaneous dialysis using the ultrafiltration system Vladisart (Russia); the pore sizes of the membrane filters were $300 \mathrm{kDa}$ and $100 \mathrm{kDa}$. The separation continued until a complete absence of carbohydrates, controlled by the phenol-sulfuric acid method.

(12) The concentrated solution was frozen at the temperature of $-40^{\circ} \mathrm{C}$ for $10-20 \mathrm{~min}$ (Chirst CB 18-40) and freeze-dried at the temperature of $-30-40^{\circ} \mathrm{C}$ (Chirst BETA 2-8 LO plus). The yields of the fractions were estimated as a percentage of the weight of the dry callus biomass. The pectin fractions with molecular weights of more than $300 \mathrm{kD}$ were used in the work.

\section{General experimental conditions}

The total content of carbohydrates in the extracts was determined by the reaction with phenol in the presence of concentrated $\mathrm{H}_{2} \mathrm{SO}_{4}$; $\left.{ }^{(12}\right)$ the content of glycuronic acids was determined by its reaction with 3,5-dimethylphenol in the presence of concentrated $\mathrm{H}_{2} \mathrm{SO}_{4}{ }^{(13)}$ (the calibration curve was plotted for D-galacturonic acid; photocolorimetry was carried out at two wavelengths $400 \mathrm{~nm}$ and $450 \mathrm{~nm}$ ). The protein content was determined by the Lowry protein assay ${ }^{(14)}$ (the calibration curve of bovine serum albumin; photocolorimetry at $750 \mathrm{~nm}$ ). Spectrophotometric measurements were performed with a SF-103 spectrophotometer (Aquilon, Russia).

\section{Total acid hydrolysis}

A $0.5 \mathrm{ml}$ of $2 \mathrm{M}$ trifluoroacetic acid (TFA) containing myo-inositol $(0.5 \mathrm{mg} / \mathrm{ml})$ was added to a sample $(1-2.5 \mathrm{mg})$. The mixture was thermostated for 4 hours at $100^{\circ} \mathrm{C}$. Excess acid was removed by repeatedly evaporating the hydrolyzate to dryness with methanol. Neutral monosaccharides were identified by the GLC method in the form of the corresponding polyol acetates.

GLC was performed on a chromatograph HewlettPackard 4890A (USA) with a flame ionization detector and an integrator HP-3395A on a capillary column RTX-1 (0.25 $\mathrm{mm} \varnothing \times 30 \mathrm{~m}$; Restek, USA); helium was a carrier gas. The temperature program ranged from $175^{\circ} \mathrm{C}(1 \mathrm{~min})$ to $250^{\circ} \mathrm{C}$ ( $2 \mathrm{~min}$ ) with the temperature of $3^{\circ} \mathrm{C} / \mathrm{min}$. The percentage of monosaccharides of the total mass of the sample was calculated from the peak areas using the detector response coefficients. ${ }^{(15)}$ Myo-inositol was an internal standard.

\section{Preparation of HPGPS}

HPGPs were obtained in the presence of calcium ions by the ionotropic gelling method 9 (Fig. 1). ${ }^{(4,16)}$

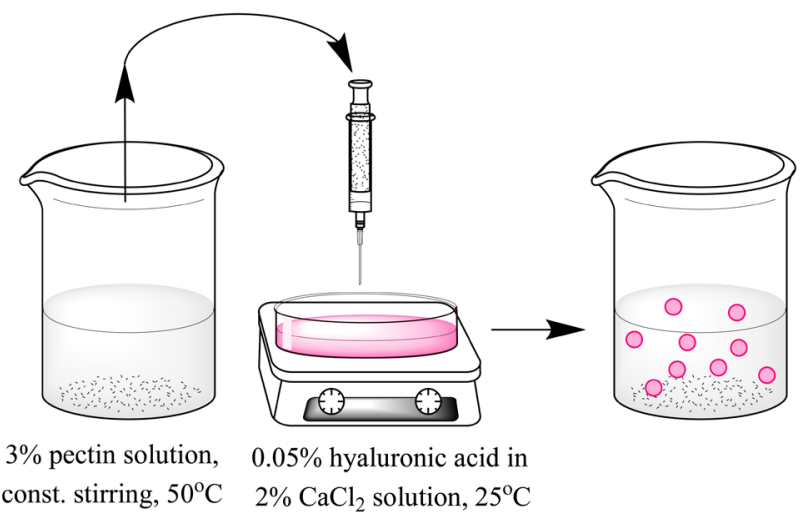

Fig. 1. Scheme for the preparation of HPGPS.

Pectins (30 mg or $50 \mathrm{mg}$ ) were dissolved in distilled water $(1 \mathrm{ml})$ by slowly stirring with a magnetic stirrer MM-5 (Russia) for 2-5 hours at room temperature until complete dissolution.

Gel particles of a spherical shape were prepared by squeezing out a solution of pectin (3\% or $5 \%$ ) from a syringe through a needle with a hole diameter of $0.6 \mathrm{~mm}$ at a distance of $4-5 \mathrm{~cm}$ in a slowly stirred $0.05 \%$ solution of HA containing calcium chloride (2\%) and further mixed for 20 minutes at room temperature. The resulting spherical gel particles were 
then washed three times in distilled water, with stirring, for 5 minutes and dried for $10-14$ hours at $37^{\circ} \mathrm{C}$. Then, the diameter, density and volume of the HPGPs were determined using an optical microscope (Altami, Russia) with a camera and an image analysis program (ImageJ 1.46r, National Institutes of Health, USA). For calibration, a linear scale was used; one pixel corresponded to $0.024 \mathrm{~mm}$.

\section{The study of the swelling and degradation of HPGPS}

To study the swelling and degradation of HPGPs under conditions simulating the gastroenteral environment, we used an artificial gastric medium (SGF solution, $\mathrm{pH} 1.25$ ), a medium of the small intestine (SIF solution, $\mathrm{pH}$ 7.0) and a medium of the large intestine (SIF solution, $\mathrm{pH} 7.0+$ pectinase (1.18 U/mg; Sigma), as described previously. ${ }^{(17)}$ Ten mg of dry gel particles of each pectin type were sequentially incubated in $5 \mathrm{ml}$ of SGF (2 h), SIF (4 h) and SIF+pectinase $(0.5,15,18$ h), with shaking (Titramax 1000, Heidolph, Germany), at 100 $\mathrm{rpm}$ and at $37^{\circ} \mathrm{C}$. At certain intervals, the diameter, density and volume of 100 randomly selected gel particles of each pectin type were determined as described above. The experiments were performed in triplicate

The degree of swelling of the gel (SD, \%) was determined by the equation;

$S D=\left(D_{1}-D_{0}\right) / D_{0} \times 100 \%$, where

$D_{1}$ - particle diameter $(\mathrm{mm})$ after a certain incubation time in the medium,

$D_{0}$ - initial particle diameter $(\mathrm{mm}){ }^{(18)}$

The statistical analysis was performed using the statistical software BioStat (version 4.03) and Microsoft Office Excel 2007. The mean (M) and standard deviation (SD) were calculated. Multiple comparisons were performed with one-way ANOVA and Tukey's HSD test. A probability value of $\mathrm{P}<0.05$ was considered statistically significant.

\section{Results and Discussion}

Pectin polysaccharides with molecular weights of more than $300 \mathrm{kDa}$ were isolated from callus cultures of TVC, LMC, SVC; their total chemical characteristic was described.

The fraction of pectin with molecular mass of more than $300 \mathrm{kDa}$ from the callus of campion had the largest yield $(2.8 \%)$. The yield of $\mathrm{SVC}>300$ was on average 2.5 times higher than the yields of $\mathrm{LMC}>300$ and TVC $>300$.

The study of the pectin monosaccharide composition revealed that residues of D-galacturonic acid, arabinose and rhamnose were the dominant constituents of all the pectins obtained. The residues of glucose, xylose, mannose and apiose were also found in the composition of fractions in a smaller amount.

Fractions of pectin with a molecular weight of more than $300 \mathrm{kDa}$ from all the cultures had a close content of D-galacturonic acid residues (75-84\%), but the ratio of galactose:arabinose was different: $1: 1.2 ; 1: 1.4$ and $1: 1.5$ for $\mathrm{LMC}>300, \mathrm{TVC}>300$ and $\mathrm{SVC}>300$, respectively. The content of galactose and arabinose residues was similar in the pectins of campion and tansy, whereas the duckweed pectin had high content of these monosaccharide residues. The sum of neutral monosaccharide residues in $\mathrm{LMC}>300(33 \%)$ was higher than in $\mathrm{SVC}>300(6 \%)$ and $\mathrm{TVC}>300(5 \%)$. The protein content of the pectin fractions was $3.0 \%(\mathrm{SVC}>300), 4.5 \%$ (TVC $>300)$ and $7.2 \%(\mathrm{LMC}>300)$

Spherical HPGPs are formed as a result of gelling, in which intermolecular cross-links arise between divalent calcium ions and negatively charged carboxyl groups of pectic macromolecules (Fig. 2) and, probably, with HA molecules. ${ }^{(19,20)}$

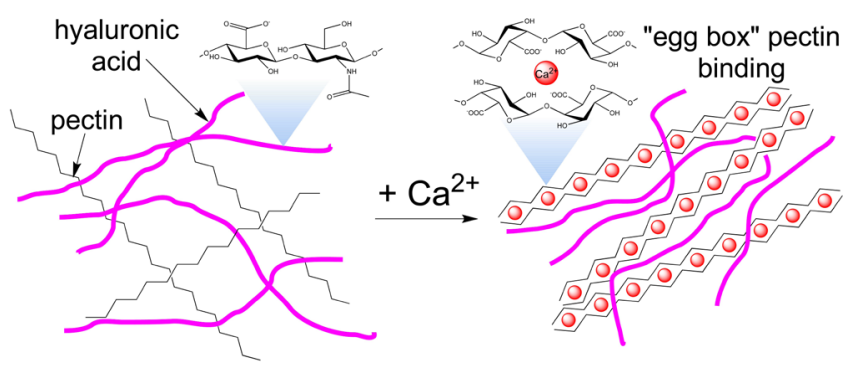

Fig. 2. Illustration of $\mathrm{Ca}^{2+}$-induced gel formation of pectic molecules in the presence of $H A$.

Previously, we studied the gelation of HA with various pectins in the presence of calcium chloride at different concentrations and tested the gelation of pectin concentrations from $0.1 \%$ to $5.0 \%$, HA from $0.01 \%$ to $2.0 \%$, and $\mathrm{CaCl}_{2}$ from 0.1 to $2.0 \%$. All pectins and HA used in the work had a molecular weight above $300 \mathrm{kD}$. It was found that the most effective gelation occurred at the pectin concentrations of $3 \%$ or $5 \%$, HA concentration of $0.05 \%$, and $\mathrm{CaCl}_{2}$ concentration of $2.0 \%$.

The formation and morphology of gel particles can be influenced by various parameters. Earlier it was shown that the concentration of HA $1 \mathrm{~g} / 1$ is minimal for obtaining hyaluronic gel particles, and the concentration of HA $6 \mathrm{~g} / 1$ leads to solutions that are too viscous. Between these boundaries, the concentration of HA does not have a significant effect on the morphology of gel particles. They have a spherical shape with a smooth surface. Gel particles obtained from pectic polysaccharides have a spherical shape. We have established that all tested hyaluronan-pectic complexes also form spherical gel particles.

The morphological characteristics of HPGPs obtained by us are presented in Table 1 . The diameters of dry HPGPs obtained on the basis of pectins AP, LMC, SVC and TVC were $1.35 \pm 0.11 \mathrm{~mm}, 0.98 \pm 0.03 \mathrm{~mm}, 0.93 \pm 0.04 \mathrm{~mm}$ and $0.83 \pm 0.04 \mathrm{~mm}$, respectively. Thus, the largest gel particles were formed in the variant with apple pectin. Similar patterns were also observed with respect to surface area and volume of HPGPs. Other researchers have obtained hyaluronic gel particles with a diameter from $8.8 \mu \mathrm{m}$ to $28.1 \mu \mathrm{m}$. ${ }^{(22)} \mathrm{S}$. Lim and colleagues ${ }^{(21)}$ obtained gel particles from HA, from chitosan and from a complex of HA with chitosan with average dimensions of $19.91 \pm 1.57 \mu \mathrm{m}, 29.47 \pm 3.58 \mu \mathrm{m}$ and $28.60 \pm 1.34$ $\mu \mathrm{m}$, respectively $(\mathrm{P}<0.05)$. That is, HPGPs we have obtained are larger than the particles from HA, from chitosan or from the complex of HA with chitosan. 
Table 1.

Morphological characteristics of dry HPGPS

\begin{tabular}{|c|c|c|c|c|}
\hline $\begin{array}{c}\text { Gel } \\
\text { particles }\end{array}$ & $\begin{array}{l}\text { Diameter, } \\
\mathrm{mm}\end{array}$ & $\begin{array}{l}\text { Area surface, } \\
\mathrm{mm}^{2}\end{array}$ & $\begin{array}{l}\text { Volume, } \\
\mathrm{mm}^{3}\end{array}$ & $\begin{array}{l}\text { Density, } \\
\mathrm{mg} / \mathrm{mm}^{3}\end{array}$ \\
\hline $\mathrm{HA}+\mathrm{AP}(1)$ & $1.35 \pm 0.11$ & $5.80 \pm 0.91$ & $1.32 \pm 0.31$ & $0.48 \pm 0.13$ \\
\hline $\mathrm{HA}+\mathrm{LMC}(2)$ & $0.98 \pm 0.03$ & $3.04 \pm 0.16$ & $0.50 \pm 0.04$ & $0.92 \pm 0.07$ \\
\hline $\mathrm{HA}+\mathrm{SVC}(3)$ & $0.93 \pm 0.04$ & $2.67 \pm 0.21$ & $0.42 \pm 0.05$ & $0.83 \pm 0.09$ \\
\hline $\mathrm{HA}+\mathrm{TVC}(4)$ & $0.83 \pm 0.04$ & $2.18 \pm 0.21$ & $0.30 \pm 0.04$ & $1.66 \pm 0.28$ \\
\hline Statistics & $\begin{array}{l}\mathrm{F}=1273.0453 \mathrm{P}=0.0000 \\
\mathrm{P}_{1-2}=0.0000 \mathrm{P}_{1-3}=0.0000 \\
\mathrm{P}_{1-4}=0.0000 \mathrm{P}_{2-3}=0.0000 \\
\mathrm{P}_{2-4}=0.0000 \mathrm{P}_{3-4}=0.0000\end{array}$ & $\begin{array}{l}\mathrm{F}=1119.5633 \mathrm{P}=0.0000 \\
\mathrm{P}_{1-2}=0.0000 \mathrm{P}_{1-3}=0.0000 \\
\mathrm{P}_{1-4}=0.0000 \mathrm{P}^{2-3}=0.0000 \\
\mathrm{P}_{2-4}=0.0000 \mathrm{P}_{3-4}=0.0000\end{array}$ & $\begin{aligned} \mathrm{F} & =845.9725 \mathrm{P}=0.0000 \\
\mathrm{P}_{1-2} & =0.0000 \mathrm{P}_{1-3}=0.0000 \\
\mathrm{P}^{1-4} & =0.0000 \mathrm{P}^{1-3}=0.0024 \\
\mathrm{P}_{2-4} & =0.0000 \mathrm{P}_{3-4}=0.0000\end{aligned}$ & $\begin{aligned} \mathrm{F} & =908.9258 \mathrm{P}=0.0000 \\
\mathrm{P}_{1-2} & =0.0000 \mathrm{P}_{1-3}=0.0000 \\
\mathrm{P}_{1-4} & =0.0000 \mathrm{P}_{2-3}=0.0007 \\
\mathrm{P}_{2-4} & =0.0000 \mathrm{P}_{3-4}=0.0000\end{aligned}$ \\
\hline
\end{tabular}

With respect to the density of HPGPs, an opposite pattern was observed. The highest density was found in HPGPs prepared on the basis of the tansy pectin TVC, and the lowest density was found for the gel particles obtained on the basis of apple pectin AP .

In sum, the largest HPGPs were formed from HA and apple pectin AP, and the densest gel particles were from HA and pectin of callus culture of tansy TVC.The swelling and degradation of pectic particles and HPGPs under conditions of an artificial environment of GIT have been studied. Gel particles derived from pectins (control) and pectins in combination with HA (experiment) were characterized by the degree of swelling of the gel (SD). The gel particles based on apple pectin were completely degraded in the SIF medium (control and experiment). Particles from the duckweed pectin gel were degraded in the SIF+P incubation medium in the control, whereas in the experiment they were degraded in combination with $\mathrm{HA}$ only in the SIF+P medium after 30 minutes. The particles of hyaluronan-pectin gels, obtained on the basis of pectins of callus cultures of campion and duckweed, did not differ significantly in the degree of swelling. It has been found that only particles of hyaluronan-pectic gels formed on the basis of the pectin of the tansy callus culture are less prone to biodegradation and have the greatest resistance in the artificial GIT as compared to other tested pectins; they do not completely break down even after 24 hours of incubation in the SIF+P medium (Fig. 3).

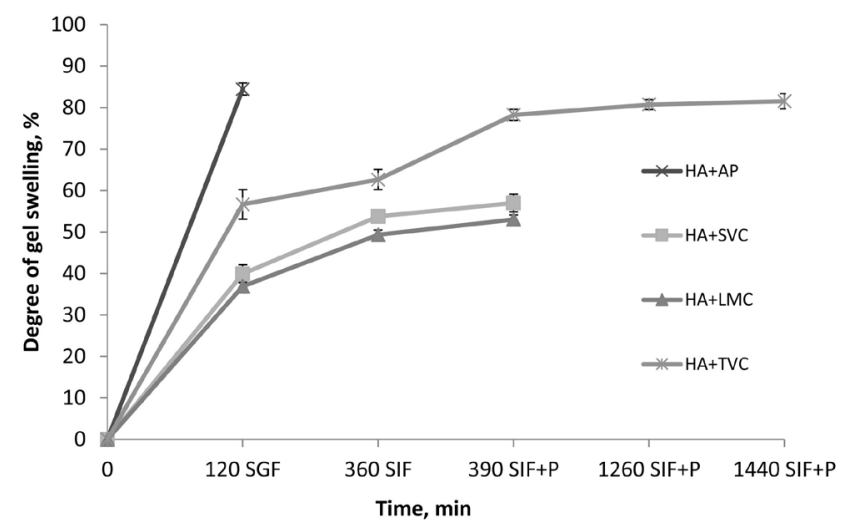

Fig. 3. Swelling and degradation of HPGPs under conditions of the artificial environment of GIT.
M. Fatnassi and colleagues showed that the hyaluronic gel particles they studied during incubation in the Tris buffer ( $\mathrm{pH}$ 7.4) were completely degraded after 22 hours of incubation.

HPGPs formed on the basis of tanacetan - tansy callus culture pectin - have fairly high stability under conditions of the artificial environment of GIT. It can be assumed that the differences in the stability of HPGPs formed from different pectins are associated with differences in molecular sizes and in the fine structure of pectic macromolecules..$^{(1,2)}$

The obtained data may indicate the prospect of further studies of the properties of hyaluronan-pectic gels, the microparticles of which can be tested as directed drug delivery systems in the small and large intestine.

\section{Competing interests}

The authors declare that they have no competing interests.

\section{References}

1. Patova EA, Golovchenko VV, Ovodov YuS. [Pectic polysaccharides: structure, properties]. Izv AN Ser Chem. 2014;9:1901-25. [Article in Russian].

2. Günter EA, Popeyko OV. Calcium pectinate gel beads obtained from callus cultures pectins as promising systems for colon-targeted drug delivery. Carbohydr Polym. 2016;147:490-9. doi: 10.1016/j.carbpol.2016.04.026

3. Günter EA, Popeyko OV, Markov PA, Martinson EA, Litvinets SG, Durnev EA, et al. Swelling and morphology of calcium pectinate gel beads obtained from Silene vulgaris callus modified pectins. Carbohydr Polym. 2014;103:550-7. doi: 10.1016.j.carbpol.2013.12.071

4. Sriamornsak P, Nunthanid J. Calcium pectinate gel beads for controlled release drug delivery: I. Preparation and in vitro release studies. Int J Pharm. 1998;160:207-12. doi: PII S03785173(97)00310-4

5. Sufiarov IF. [Experimental substantiation of the use of a film based on modified hyaluronic acid to prevent the formation of postoperative peritoneal adhesions]. Bull Exp Biol Med. 2007;144(8):238-40. [Article in Russian].

6. Uspensky SA, Kildeeva NR, Maslova MV, Demina TS, Vikhoreva GA.[ Investigation of the stability of viscous properties of hyaluronic acid solutions for the preparation of 
polyelectrolyte complexes with chitosan]. Izv AN Ser Chem. 2016;1:273-76. [Article in Russian].

7. Zhou Z, He S, Huang T, Peng C, Zhou H, Liu Q, et al. Preparation of gelatin/hyaluronic acid microspheres with different morphologies for drug delivery. Polym Bull. 2015;72:713-23. doi: 10.1007/s00289-015-1300-0

8. Vildanova RR, Sigaeva NN, Kukovinets OS, Vlasova NM, Kolesov SV. [Modified hyaluronic acid and chitosan for production of hydrogels]. Vestnik Bashkirskogo Universiteta. 2016;21(1):63-68. [Article in Russian].

9. Murashige T, Skoog. F. A revised medium for rapid growth and bio assays with tobacco tissue cultures. Physiologia Plantarum. 1962;15(3):473-97. doi: 10.1111/ j.1399-3054.1962.tb08052.x

10. Günter EA. [Preparation of callus cultures of Silene vulgaris (M.) G.]. Biotechnology. 2002;6:41-45. [Article in Russian].

11. Günter EA, Popeyko OV, Ovodov YuS. [Isolation of polysaccharides from the callus culture of Lemna minor L.]. Prikl Biokhim Microbiol. 2004;40(1):94-97. [Article in Russian].

12. Dubois M, Gilles KA, Hamilton JK, Rebers PA, Smith F. Colorimetric method for determination of sugars and related substances. Analyt Chem. 1956;28(3):350-56.

13. Usov AI, Bilan MI, Klochkova NG. Polysaccharide composition of several calcareous red algae: isolation of alginate from Corallina pilulifera P. et R. (Rhodophyta, Corallinaceae). Bot Marina. 1995;38:43-51. doi: 10.1515/ botm.1995.38.1-6.43

14. Lowry OH, Rosebrough NJ, Farr AL, Randall RJ. Protein measurement with the Folin phenol reagent. J Biol Chem. 1951;193:265-75.

15. York WS, Darvill AG, McNeil M, Stevenson TT,
Albercheim P. Isolation and characterization of plant cell walls and cell-wall components. Methods Enzymol. 1985;118:3-40. 16. Sriamornsak P. Effect of calcium concentration, hardening agent and drying condition on release characteristics of oral proteins from calcium pectinate gel beads. Eur J Pharm Sci. 1999;8(3):221-7.

17. Gebara C, Chaves KS, Ribeiro MCE, Souza FN, Grosso CRF, Gigante ML. Viability of Lactobacillus acidophilus La5 in pectin-whey protein microparticles during exposure to simulated gastrointestinal conditions. Food Res Int. 2013;51:872-78. doi: 10.1016/j.foodres.2013.02.008

18. Oliveira GF, Ferrari PC, Carvalho LQ, Evangelista RC. Chitosan-pectin multiparticulate systems associated with enteric polymers for colonic drug delivery. Carbohydr Polym. 2010;82(3):1004-09. doi: 10.1016/j.carbpol.2010.06.041

19. Chambin O, Dupuis G, Champion D, Voilley A, Pourcelot Y. Colon-specific drug delivery: Influence of solution reticulation properties upon pectin beads performance. Int $\mathrm{J}$ Pharm. 2006;321(1-2):86-93.

20. Sriamornsak P, Kennedy RA. Swelling and diffusion studies of calcium polysaccharide gels intended for film coating. Int J Pharm. 2008;358(1-2):205-13. doi: 10.1016/j. ijpharm.2008.03.009.

21. Lim ST, Martin GP, Berry DJ, Brown MB. Preparation and evaluation of the in vitro drug release properties and mucoadhesion of novel microspheres of hyaluronic acid and chitosan. J Control Release. 2000;66(2-3):281-92.

22. Fatnassi M, Jacquart S, Brouillet F, Rey C, Combes C, Fullana SG. Optimization of spray-dried hyaluronic acid microspheres to formulate drug-loaded bone substitute materials. Powder Technol. 2014; 255:44-51. doi: 10.1016/j. powtec.2013.08.027. 